\title{
Interview Report
}

How do we assess the validity of social science findings pertaining to countries and contexts we might know nothing about? In an era of fake news, and increasingly deep fakes as well that build on a long history of stereotype we might not even be aware we subscribe to, it seems helpful to show readers what this book is based on. Later on I explain the methodology more comprehensively, but here I show the formal interviews. These were discussions that happened, that were recorded, on these places, on these days, and they form the skeleton around which the book has emerged.

\begin{tabular}{ccc}
\hline No. & Place & Date \\
\hline 1 & Lobito & 131106 \\
2 & Lobito & 131112 \\
3 & Lobito & 131112 \\
4 & Lobito & 131119 \\
5 & Lobito & 131120 \\
6 & Lobito & 131121 \\
7 & Lobito & 131122 \\
8 & Lobito & 131124 \\
9 & Lobito & 131202 \\
10 & Lobito & 131210 \\
& & (continued)
\end{tabular}




\begin{tabular}{|c|c|c|}
\hline No. & Place & Date \\
\hline 11 & Lobito & 131211 \\
\hline 12 & Benguela & 131212 \\
\hline 13 & Lobito & 131212 \\
\hline 14 & Benguela & 131213 \\
\hline 15 & Lobito & 131216 \\
\hline 16 & Lobito & 131217 \\
\hline 17 & Lobito & 131217 \\
\hline 18 & Lobito & 131217 \\
\hline 19 & Lobito & 131218 \\
\hline 20 & Lobito & 131220 \\
\hline 21 & Lobito & 131230 \\
\hline 22 & Benguela & 140108 \\
\hline 23 & Lobito & 140109 \\
\hline 24 & Lobito & 140110 \\
\hline 25 & Lobito & 140112 \\
\hline 26 & Lobito & 140116 \\
\hline 27 & Lobito & 140117 \\
\hline 28 & Lobito & 140118 \\
\hline 29 & Lobito & 140207 \\
\hline 30 & Benguela & 140216 \\
\hline 31 & Catumbela & 140221 \\
\hline 32 & Lobito & 140224 \\
\hline 33 & Benguela & 140302 \\
\hline 34 & Lobito & 140308 \\
\hline 35 & Lobito & 140309 \\
\hline 36 & Benguela & 140314 \\
\hline 37 & Lobito & 140318 \\
\hline 38 & Lobito & 140321 \\
\hline 39 & Lobito & 140326 \\
\hline 40 & Lobito & 140327 \\
\hline 41 & Lobito & 140328 \\
\hline 42 & Lobito & 140402 \\
\hline 43 & Lobito & 140403 \\
\hline 44 & Catumbela & 140424 \\
\hline 45 & Lobito & 140425 \\
\hline 46 & Benguela & 140503 \\
\hline
\end{tabular}




\begin{tabular}{|c|c|c|}
\hline No. & Place & Date \\
\hline 47 & Lobito & 140507 \\
\hline 48 & Lobito & 140513 \\
\hline 49 & Lobito & 140515 \\
\hline 50 & Lobito & 140519 \\
\hline 51 & Lobito & 140521 \\
\hline 52 & Lobito & 140522 \\
\hline 53 & Lobito & 140526 \\
\hline 54 & Luanda & 140527 \\
\hline 55 & Luanda & 140527 \\
\hline 56 & Benguela & 140528 \\
\hline 57 & Lobito & 140603 \\
\hline 58 & Lobito & 140603 \\
\hline 59 & Lobito & 140605 \\
\hline 60 & Lobito & 140606 \\
\hline 61 & Lobito & 140606 \\
\hline 62 & Rio de Janeiro & 140729 \\
\hline 63 & Rio de Janeiro & 140821 \\
\hline 64 & Rio de Janeiro & 140823 \\
\hline 65 & Rio de Janeiro & 140824 \\
\hline 66 & Rio de Janeiro & 140828 \\
\hline 67 & Rio de Janeiro & 140829 \\
\hline 68 & Rio de Janeiro & 140905 \\
\hline 69 & Rio de Janeiro & 140907 \\
\hline 70 & Rio de Janeiro & 140908 \\
\hline 71 & Rio de Janeiro & 140910 \\
\hline 72 & Rio de Janeiro & 140911 \\
\hline 73 & Rio de Janeiro & 140912 \\
\hline 74 & Rio de Janeiro & 140918 \\
\hline 75 & Rio de Janeiro & 140921 \\
\hline 76 & Rio de Janeiro & 140922 \\
\hline 77 & Rio de Janeiro & 140922 \\
\hline 78 & Rio de Janeiro & 140923 \\
\hline 79 & Rio de Janeiro & 140926 \\
\hline 80 & Juiz da Fora & 140927 \\
\hline 81 & Rio das Ostras & 140929 \\
\hline 82 & Rio de Janeiro & 140930 \\
\hline
\end{tabular}




\begin{tabular}{|c|c|c|}
\hline No. & Place & Date \\
\hline 83 & Rio de Janeiro & 141001 \\
\hline 84 & Rio de Janeiro & 141002 \\
\hline 85 & Rio de Janeiro & 141002 \\
\hline 86 & Niteroí & 141003 \\
\hline 87 & Rio de Janeiro & 141004 \\
\hline 88 & Rio de Janeiro & 141008 \\
\hline 89 & Rio de Janeiro & 141010 \\
\hline 90 & Rio de Janeiro & 141016 \\
\hline 91 & Curitiba & 141018 \\
\hline 92 & Curitiba & 141019 \\
\hline 93 & Curitiba & 141020 \\
\hline 94 & Curitiba & 141021 \\
\hline 95 & Curitiba & 141021 \\
\hline 96 & Curitiba & 141022 \\
\hline 97 & Curitiba & 141023 \\
\hline 98 & Rio de Janeiro & 141030 \\
\hline 99 & Rio de Janeiro & 141030 \\
\hline 100 & Rio de Janeiro & 141030 \\
\hline 101 & Rio de Janeiro & 141031 \\
\hline 102 & Rio de Janeiro & 141106 \\
\hline 103 & Rio de Janeiro & 141110 \\
\hline 104 & Rio de Janeiro & 141110 \\
\hline 105 & Lobito & 141209 \\
\hline 106 & Lobito & 141211 \\
\hline 107 & Lobito & 180514 \\
\hline
\end{tabular}

